\title{
Imposing minimax and quantile constraints on optimal matching in observational studies SOFTWARE NOTES
}

\author{
Paul R. Rosenbaum ${ }^{1}$, University of Pennsylvania
}

The paper proposes a method for imposing a minimax constraint or a quantile constraint or a sequence of such contraints on an optimal match obtained by minimizing the total cost of a flow in a network. This additional constraint could be added to many different types of matched samples, and to several existing software packages.

To illustrate a simple version of the technique, I have written a simple automatic matching package in $\mathrm{R}$ called aamatch. Versions of this package are included with this manuscript submission.

The aamatch function in the aamatch package implements the idea in $\S 2.5$ of the manuscript. Specifically, aamatch forces fine or near-fine balance on the propensity score, then adds the constraint that the maximum within-pair robust Mahalanobis distance is minimized, then subject to those two constraints, it minimizes a total within-pair penalized covariate distance.

The example in the help file for the matchfine3 function creates "match $4 "$ in the paper. Match 4 has various features specific to the example in the current paper.

\footnotetext{
${ }^{1}$ Department of Statistics, The Wharton School, University of Pennsylvania, Philadelphia, PA 19104. rosenbaum @wharton.upenn.edu. 22 July 2015. Supported by the US National Science Foundation.
} 